\title{
El Rol del Docente de Matemáticas en Educación Virtual Universitaria. Un Estudio en la Universidad Pedagógica y Tecnológica de Colombia
}

\author{
Hugo H. Mendoza ${ }^{(1) \star}$, Víctor M. Burbano(2), Margoth A. Valdivieso(3) \\ (1) Univ. Pedagógica y Tecnológica de Colombia, Uptc. Av Central del Norte, Tunja Colombia. \\ (e-mail: 2hsmendoza@gmail.com) \\ (2) Facultad de Ciencias, Escuela de matemáticas, Univ. Pedagógica y Tecnológica de Colombia, Uptc. Av Central del \\ Norte, Tunja Colombia. (e-mail: victor.burbano@uptc.edu.co) \\ (3) Escuela de matemáticas, Univ. Pedagógica y Tecnológica de Colombia, Uptc. Av Central del Norte, Tunja Colombia. \\ (e-mail: margoth.valdivieso@uptc.edu.co)
}

* Autor a quien debe ser dirigida la correspondencia

Recibido Ene. 21, 2019; Aceptado Mar. 18, 2019; Versión final Abr. 25, 2019, Publicado Oct. 2019

\begin{abstract}
Resumen
En este artículo se indaga sobre el rol del tutor de matemáticas que trabaja en el programa de ciencias tecnológicas, modalidad virtual de la Facultad de Estudios a Distancia (FESAD) de la Universidad Pedagógica y Tecnológica de Colombia (UPTC). En esta investigación se utilizó un enfoque cuantitativo de corte exploratorio, la muestra estuvo conformada por 21 docentes-tutores del mencionado programa a quienes se les aplicó una encuesta. Los datos fueron procesados por medio del software libre R. Los resultados permitieron establecer que, el tutor de matemáticas utiliza didácticas específicas orientadas a la construcción de conceptos, medios y mediaciones focalizadas en las TICs y formas de evaluación propias del modelo virtual. Un $76.1 \%$ se caracteriza por ser mediador y moderador durante el proceso enseñanza aprendizaje. Se concluye que, el tutor está moderadamente permeado por los ambientes virtuales y por algunas estrategias asociadas con las didácticas específicas para la enseñanza de la matemática.
\end{abstract}

\section{The Role of the Teacher of Mathematics in Virtual University Education. A Study in the Pedagogic and Technologic University of Colombia}

\begin{abstract}
This article explores the role of the tutor of mathematics working in the program of technological sciences, virtual modality of the Faculty of Distance Studies (FESAD) of the Pedagogical and Technological University of Colombia (UPTC). In this research, a quantitative approach of exploratory cut was used, the sample consisted of 21 teachers-tutors of the mentioned program to whom a survey was applied. The data was processed by means of the free software $R$. The results allowed establishing that the tutor of mathematics uses specific didactics aimed at the construction of concepts, media and mediations focused on ICT and forms of evaluation of the virtual model. Of the sample, $76.1 \%$ is characterized as a mediator and moderator during the teaching-learning process. It is concluded that the tutor is moderately permeated by virtual environments and by some strategies associated with specific didactics for the teaching of mathematics.
\end{abstract}

Keywords: role of the tutor; virtual model; mathematics didactics; ICTS 


\section{INTRODUCCIÓN}

Diversos estudios plantean la necesidad de promover mejoras en la calidad educativa; un elemento que influye sobre ésta es el rol desempeñado por el docente, debido a que es quien tiene la tarea de desarrollar el currículo y ser un guía en los procesos de aprendizaje (Burbano et al., 2017); en este sentido, Marciniak y Sallán (2018) mencionan que, el rol del docente está asociado con el logro académico estudiantil y el docentetutor es quien ha de orientar el trabajo de los estudiantes, con diversas actividades formativas a través de metodologías activas y participativas en diferentes modelos educativos, entre ellos, el virtual. De acuerdo con García (1999), la educación virtual (EV) es un sistema tecnológico de comunicación bidireccional, caracterizado por ser masivo, basado en la acción sistemática y conjunta de recursos didácticos y el apoyo de una organización y tutoría que, separados físicamente del estudiante, propicia en él un aprendizaje independiente, flexible y autónomo. Este sistema promueve el manejo de espacios y tiempos, estilos y ritmos de aprendizaje (Barberá, 2000), teniendo en cuenta las individualidades y la interacción con los otros, en un entorno tecnológico, cultural y social (Martínez et al., 2018); no se trata de una instrumentación técnica para adaptar contenidos e impartirlos por medios electrónicos; sino de un modelo pedagógico virtual para compartir conocimientos e implementar acciones tutoriales centradas en didácticas específicas y mediadas por las TIC con el fin de mejorar aspectos de cobertura, de adquisición del conocimiento y de superar la presencia física en el salón de clases tradicional (Hernández, 2012).

Además, la EV favorece a los estudiantes, en el sentido que, no tienen que renunciar a su trabajo para dedicarse a estudiar; ni tampoco dejar incompleta su formación académica por abandonar los estudios, ya que las TIC se han extendido de forma remota más allá del aula tradicional (ChanLin, 2016); también, la EV les permite realizar sus estudios aun viviendo en lugares muy distantes, sin tener que cambiarse de residencia para poder asistir a clases, manejar los espacios y tiempos de acuerdo a sus necesidades, la calidad de la enseñanza depende de un equipo interdisciplinario que realiza y revisa los materiales de apoyo; estos aspectos pueden disminuir los gastos educativos. En referencia a la Educación virtual en Colombia, se ha observado que: i) ésta también es conocida como educación a distancia y con el trascurso del tiempo ha sufrido diversas diferentes transformaciones, ii) los procesos educativos se dieron en diferentes contextos y el uso de variados recursos (material impreso, teleconferencia, comunicación epistolar, entre otros), y iii) los medios tecnológicos fueron determinantes para proponer alternativas metodológicas (Martínez et al., 2018). Esto significa que la forma tradicional de educación ha de cambiar sus estrategias a fin de mejorar la calidad en la enseñanza y alcanzar una mayor cobertura educativa sobre poblaciones menos favorecidas. En el modelo de EV, la relación no presencial entre tutor y estudiante no altera el logro de los objetivos de aprendizaje y permite optimizar tiempo y recursos (Silva, 2010).

Desde hace tres décadas, la UPTC ha venido desarrollando una serie de programas educativos a nivel tecnológico, como alternativa para poblaciones con algunas dificultades de tiempo y espacio en el acceso a la educación superior presencial. Actualmente, en dichos programas, los docentes hacen uso de las TIC y de herramientas como las plataformas virtuales. Con base en los informes de valoración del desempeño docente, en los cursos de matemáticas impartidos en los diferentes programas de tecnologías en la FESAD, se han evidenciado dificultades asociadas con el rol del docente-tutor en el manejo eficaz de medios y herramientas metodológicas a la hora de impartir conocimientos, de potenciar en sus estudiantes altos niveles de pensamiento lógico y competencias matemáticas, y de lograr un buen rendimiento académico. En este contexto, se formuló la siguiente pregunta de investigación ¿cuáles aspectos caracterizan al tutor de matemáticas en la escuela de ciencias tecnológicas de la FESAD? Según García (1999), la característica fundamental del tutor es la de fomentar el desarrollo del estudio independiente y de constituirse en un orientador del aprendizaje de aquel alumno aislado, solitario y carente de la presencia del docente habitual. En este sentido, la figura del tutor cobra mayor significado cuando se hace cargo de su asistencia y ayuda personal, a la vez que representa un determinado nexo con la educación virtual (Barberá, 2000); en este contexto, el tutor ha de motivar y dinamizar los grupos de trabajo, organizar actividades pertinentes, facilitar la construcción de conocimiento y fomentar el auto-aprendizaje (Silva, 2010). Estos aspectos se formalizan entre tutor y estudiante, cuando mantiene una comunicación activa durante la construcción del conocimiento.

En general, el docente virtual (tutor) debe ser un experto en la materia que imparte, sus funciones principales serán las de guiar, estimular y colaborar con el estudiante en su proceso de aprendizaje; el tutor ha de constituirse en un mediador, promotor y facilitador en la asimilación y construcción del conocimiento estudiantil, y evaluador de sus aprendizajes en relación con una asignatura o un conocimiento específico (Ernest, 1998); para ello, ha de utilizar diversas estrategias que contrasten con la pedagogía tradicional, tales como el aprendizaje autónomo, situaciones didácticas mediadas por las TIC, manejo de entornos virtuales, aprendizaje colaborativo y cooperativo en red, entre otros; es decir, el docente-tutor debe conjugar el papel de experto en contenidos con el de facilitador del aprendizaje mediante didácticas específicas (Silva, 2010). 
Marcos (2009) indica la necesidad de potenciar el aprendizaje autónomo, de modo que, se genere un cambio individual, cultural y social, pasando de entornos caracterizados por un profesor dominante (tradicional) a otros más centrados en el alumno. Además, considera que, los entornos interactivos de aprendizaje mediados por las TIC con el apoyo eficiente del tutor, generan beneficios cognitivos en los estudiantes para adquirir competencias matemáticas, siempre que se apliquen modelos didácticos pertinentes, asociados con metodologías activas y constructivas que promuevan el aprendizaje significativo. En este contexto, la acción tutorial universitaria ha de incluir un conjunto de procesos tendientes a que el alumnado adquiera tanto competencias específicas (por ejemplo, las matemáticas) como generales, entre ellas, aprender a aprender, aprender a trabajar, aprender a vivir juntos (García et al., 2018).

Adicionalmente, se considera que las TIC, son cada vez más amigables, accesibles y adaptables a la acción del tutor. De allí que tanto en el docente como en la institución educativa las pueden incorporar para generar mejoras en la acción didáctica; de ese modo, se puede transitar de la enseñanza tradicional hacia un aprendizaje más colaborativo y en red; los ambientes virtuales también pueden proporcionar información sobre los contenidos matemáticos y promover el desarrollo de habilidades y destrezas (Castro et al., 2007). Con el advenimiento de la llamada Web 2.0, los Entornos Virtuales de Aprendizaje -EVA-y video-tutoriales aparecen como tecnologías emergentes para apoyar la labor docente (Colestock y Sherin, 2009); los EVA contribuyen con la mejora de la clase tradicional, posibilitan la construcción de espacios de aprendizaje más allá de las fronteras del aula y plantean nuevas competencias y habilidades para el tutor, entre ellas, generar un diálogo efectivo con y entre los participantes, promover el aprendizaje activo, cooperativo y colaborativo, y efectuar el monitoreo y moderación de la participación estudiantil tanto del trabajo en grupo como independiente. El rol de moderador implica planificación, intervención en el desarrollo de una discusión y cierre con retroalimentación (Barberá, 2000).

En los entornos virtuales, el rol del tutor incluye tres aspectos: diseño y organización, manejo del discurso y dinamización grupal; de aquí se deduce que, el rol del tutor implica diseñar, facilitar la comunicación bidireccional (uso de medios sincrónicos y asincrónicos) y promover procesos cognitivos y sociales, con el propósito de obtener resultados significativos en el aprendizaje de una temática específica. Un rol adicional implícito en el tutor es el de lograr empatía entre quienes interactúan en el proceso enseñanza-aprendizaje a fin de generar espacios de confianza en el desarrollo de las tareas asignadas; en este sentido, Martínez et al. (2018) sugiere que el tutor ha de monitorear de forma permanente los procesos y los progresos de sus estudiantes, con la intención de que se acreciente el aprendizaje autónomo y se efectúe una parte del proceso de evaluación. Al respecto, Pagano (2008) considera que un tutor efectivo ha de poseer atributos como empatía, pro-acción, ser buen anfitrión, tener maestría comunicativa, usar metodologías no convencionales y realizar evaluación permanente. Con aspectos como las mencionadas, el tutor puede constituirse en un elemento esencial para mejorar la calidad educativa desde el modelo virtual.

Para el caso particular de la matemática, el rol del tutor ha de involucrar tres aspectos centrales: el conocimiento matemático (CM), las didácticas específicas y el manejo de entornos virtuales. En el primer aspecto se deben incorporar tanto elementos formales como concretos (Ernest, 1998); aquí, el conocimiento del lenguaje matemático y su simbología, la metamatemática de las demostraciones y definiciones, se consideran como estructuras formales (Depaepe et al., 2013); por lo tanto, el CM que ha de poseer el tutor corresponde a una combinación de los conocimientos comunes de los contenidos matemáticos y los conocimientos especializados (Ball et al., 2008). Además, el CM puede orientarse bajo una práctica pedagógica pertinente (Mason, 2002) y sustentarse en diversos modelos de conocimiento del profesor (Burbano et al., 2017). La didáctica específica ha de entenderse como la ciencia del desarrollo de las planificaciones realizables en la enseñanza de una determinada materia; en este sentido, D'Amore y Godino (2006) sostienen que, ésta es una parte de las ciencias de la educación destinada a la selección de programas, situaciones y estrategias didácticas, uso de manuales y materiales de apoyo asociados con los métodos de enseñanza. Además, la didáctica estudia la dinámica del proceso enseñanza-aprendizaje con sus relaciones dialécticas, individuales y grupales (Abreu et al., 2018); este proceso puede verse como un sistema conformado por el saber, el profesor, el estudiante (Chevallard, 1999) y el contexto, el cual puede incluir un entorno virtual con efecto contrario a la clásica transmisión de saberes (Silva, 2010), enmarcado en la perspectiva teórica de la trasposición didáctica que trasciende la mera postura psicológica e integra una mirada antropológica y epistemológica del saber enseñado (Villalobos y Melo, 2019).

En la enseñanza de las matemáticas, el tutor puede apoyarse también en praxeologías a través de cuatro elementos básicos: las tareas, las técnicas (mediaciones), las tecnologías (medios) y los aspectos matemáticos teóricos (Chevallard, 1999); asimismo, en situaciones didácticas (Brousseau, 1986) diseñadas y expuestas por el tutor a fin de orientar procesos tendientes a que el estudiante construya el conocimiento matemático mediante abstracción reflexiva. Lo anterior indica que el tutor ha de tener claros los objetos matemáticos particulares por comunicar con la ayuda de diversos medios y mediaciones. Para dinamizar el aprendizaje con situaciones didácticas mediadas por las TIC, el tutor puede utilizar materiales de estudio 
digitalizados, la simulación como un recurso didáctico (Burbano et al., 2015), narrativas docentes y trasferencias didácticas (Villalobos y Melo, 2019), guías de trabajo individual y en grupo, discusiones por medio de foros, chat, videoconferencias, interacción y evaluación a través de la plataforma virtual o de contrato didáctico, entre otros.

En los dos últimos lustros, los debates giraban en torno a definir los tipos de conocimientos y la experiencia que los docentes de matemáticas deberían poseer para direccionar de mejor manera su acción didáctica (Fraser, 2015). En este sentido, Burbano et al. (2017), sugieren apoyarse en el Enfoque OntoSemiótico EOS- 0 en otros modelos de conocimiento del profesor, a fin de promover la construcción de conceptos matemáticos, haciendo uso de formas o procedimientos de orden lógico y lingüístico; el EOS puede favorecer la cognición e instrucción matemática asignándole un papel central al lenguaje, a los procesos de comunicación e interpretación y a la variedad de objetos que se ponen en juego en la enseñanza y el aprendizaje de las matemáticas. Estos autores señalan que existen siete categorías de conocimiento que podría activar el docente durante la enseñanza: conocimiento del contenido, conocimiento pedagógico general, conocimiento del currículo, conocimiento pedagógico del contenido -PCK- (Shulman, 1986), conocimiento de los estudiantes y sus características, conocimiento de los contextos educativos y conocimiento de los fines, propósitos y valores de la educación.

Finalmente, en este contexto, se espera que el tutor pueda utilizar de manera eficiente didácticas específicas pertinentes y elementos de los entornos virtuales para dinamizar el aprendizaje de las matemáticas en la FESAD, también, podría apoyarse en las fuentes del conocimiento expuestas por Shulman (1986) para mejorar su acción tutorial: suficiente formación académica, materiales pertinentes, investigación sobre la enseñanza y el aprendizaje, fenómenos socioculturales y la sabiduría que otorga la experiencia docente. Por consiguiente, el objetivo de este trabajo consistió en indagar sobre algunos aspectos que caracterizan al tutor de matemáticas en la escuela de ciencias tecnológicas de la FESAD. Además, el trabajo aporta información relevante sobre aspectos asociados con el uso de medios tecnológicos y estrategias didácticas para enseñar matemáticas a nivel superior desde la educación virtual.

\section{METODOLOGÍA}

Este estudio presenta un enfoque cuantitativo de investigación, centrado en los métodos descriptivos de corte transversal para el tratamiento de la información; de acuerdo con Hernández et al. (2003), estos métodos incluyen la recolección de datos por medio de variables tendientes a explorar determinados aspectos de interés en un grupo de individuos; además, posibilitan hacer inferencias con base en la medición numérica y el análisis estadístico, para establecer patrones de comportamiento. Por consiguiente, en esta investigación, se empleó una metodología de tipo cuantitativo por cuanto a partir de los datos observables en los cuestionarios se obtuvieron resultados tendientes a probar las hipótesis y un carácter descriptivo porque buscan especificar características específicas de los participantes en el estudio. Los individuos que conformaron el grupo de estudio fueron 21 docentes-tutores, quienes orientan la asignatura de matemáticas en el programa de ciencias tecnológicas, modalidad virtual de la Facultad de Estudios a Distancia (FESAD) de la Universidad Pedagógica y Tecnológica de Colombia (UPTC), tal asignatura se ofrece en diferentes semestres. El grupo estuvo conformado por 7 tutoras y 14 tutores; es decir, un $33.33 \%$ de los participantes fueron de género femenino y el $66.67 \%$ de género masculino. Esta conformación resultó así debido a que se tomaron todos los tutores de matemáticas contratados por la UPTC en el año 2018 para trabajar en el mencionado programa; es conveniente mencionar que, la UPTC vincula a sus docentes mediante procesos de selección por méritos académicos e investigativos.

El objetivo del estudio, la pregunta de investigación y el marco teórico permitieron plantear esta hipótesis: H1: El rol del tutor de matemáticas que trabaja en ciencias tecnológicas de la FESAD se aproxima al requerido en el modelo de educación virtual; H2: Las didácticas específicas usadas por el tutor de matemáticas en el programa ciencias tecnológicas de la FESAD están asociadas con su tiempo de trabajo en la modalidad virtual; H3: Las metodologías y las formas de evaluación utilizadas por el docente de matemáticas que trabaja en ciencias tecnológicas de la FESAD si guardan relación con el rol del tutor en el modelo de educación virtual.

Para recolectar la información se aplicó a los docentes-tutores, un cuestionario constituido por nueve preguntas asociadas con el rol del tutor en el contexto de la FESAD en la UPTC, éstas fueron: género, título profesional, años de experiencia profesional, tiempo de trabajo en la modalidad virtual en la FESAD, uso de didácticas específicas tradicionales, metodologías utilizada en el proceso de enseñanza de las matemáticas, medios y mediaciones usados con frecuencia, rol del tutor durante el proceso de enseñanza de las matemáticas y las formas de evaluación. El cuestionario fue sometido a juicio de tres expertos antes de ser aplicado, el primero en didáctica de las matemáticas, el segundo en educación virtual y el tercero en estadística. 
En este estudio, el $38 \%$ de los participantes acreditó tituló en el área de matemáticas y física, el 33.3\% en matemáticas y el $28.7 \%$ en matemáticas y estadística. Esta composición en el perfil profesional se atribuye a que en la UPTC, las dos primeras titulaciones son las más antiguas. Además, la UPTC también contribuye con la formación de profesionales en estas áreas para cubrir tanto la demanda interna como la externa, respondiendo a las exigencias actuales del currículo de matemáticas en el nivel universitario y de la educación secundaria (Stein et al., 2007) tanto en el ámbito local y regional como nacional.

El análisis de los datos se realizó a través del software libre $R$ y la hoja de cálculo de Excel y algunos elementos del paquete estadístico SPSS en su versión de prueba. Con estas herramientas se elaboraron las gráficas y se generaron porcentajes para efectuar la interpretación de los resultados.

\section{RESULTADOS Y DISCUSIÓN}

De acuerdo con Shulman (1986) la experiencia profesional docente es una fuente de conocimiento que podría influir de manera favorable en la enseñanza de diversos tópicos, en particular, en temas de matemáticas en distintos niveles y contextos educativos. En este estudio, los resultados evidencian que predominan los docentes con más de 20 años de experiencia en el área de las matemáticas o afines (38.1\%); le siguen, quienes acreditan entre 11 y 20 años (33.33\%), y solo el $28.57 \%$ presentan menos de 1 hasta 10 años, tal experiencia fue adquirida tanto en el nivel universitario como de la educación secundaria (ver Fig. 1); los docentes manifiestan que han transitado de un nivel a otro y paulatinamente han incorporado elementos tecnológicos en sus clases. Estos aspectos guardan relación con lo expuesto por Schoenfeld y Kilpatrick (2008) en el sentido que, pueden favorecer la el desempeño docente; además, posibilitan el uso de metodologías propias de la modalidad virtual (Martínez et al., 2018).

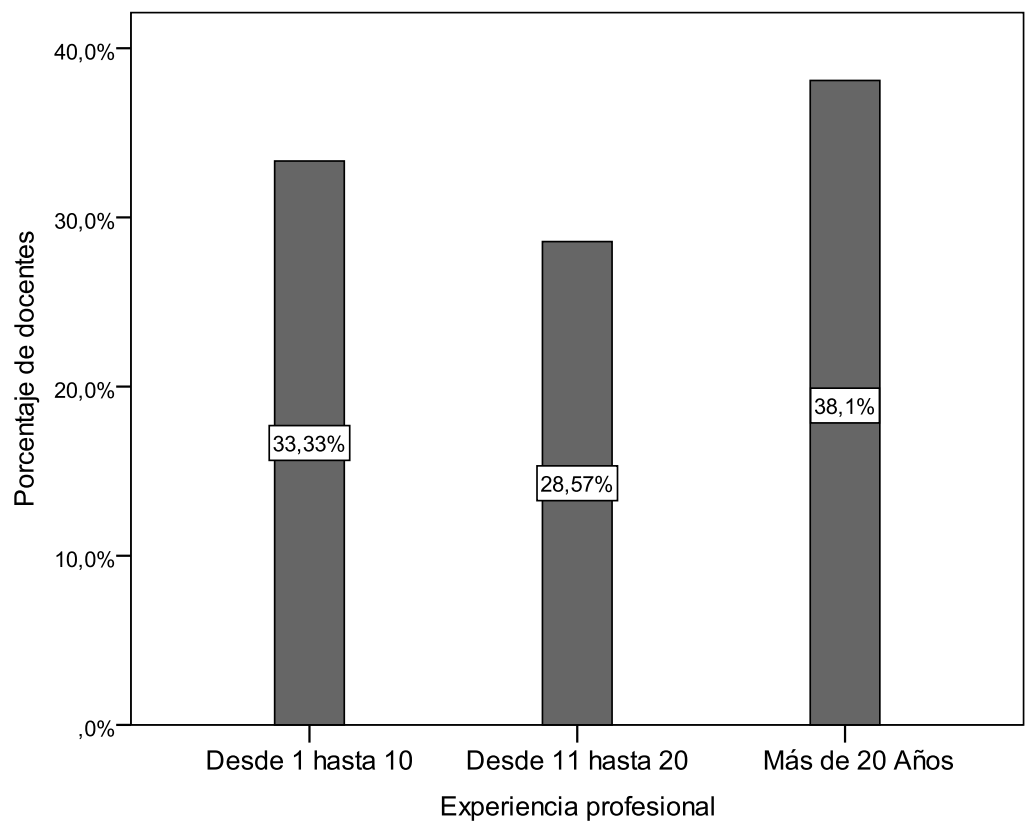

Fig. 1: Años de experiencia profesional docente

En referencia al tiempo de trabajo en la modalidad virtual en la FESAD, se establece una prevalencia de los docentes que han trabajado desde 1 hasta 10 años (47.62\%), seguida por quien tienen entre los 11 y 20 años (33.33\%), y un menor porcentaje $(19.05 \%)$ corresponde a los docentes más experimentados en la modalidad virtual (ver Fig. 2). Este estado de la cuestión se explica por la incorporación paulatina de las TIC y los ambientes virtuales en el contexto universitario, desde hace aproximadamente tres décadas.

Estos resultados concuerdan con Silva (2010), quien sostiene que, en tanto las generaciones nuevas de docentes se instruyan y asimilen esta modalidad de trabajo, apoyados con espacios de interacción virtual, se dispondrá de un talento humano mejor preparado para cumplir de manera más pertinente, con su función tutorial. Por su parte Menéndez (2012) destaca la necesidad de acrecentar las competencias docentes bajo entornos virtuales, de modo que el tutor sea un mediador efectivo en la enseñanza de un determinado tópico; en este contexto, las matemáticas no serían la excepción. 


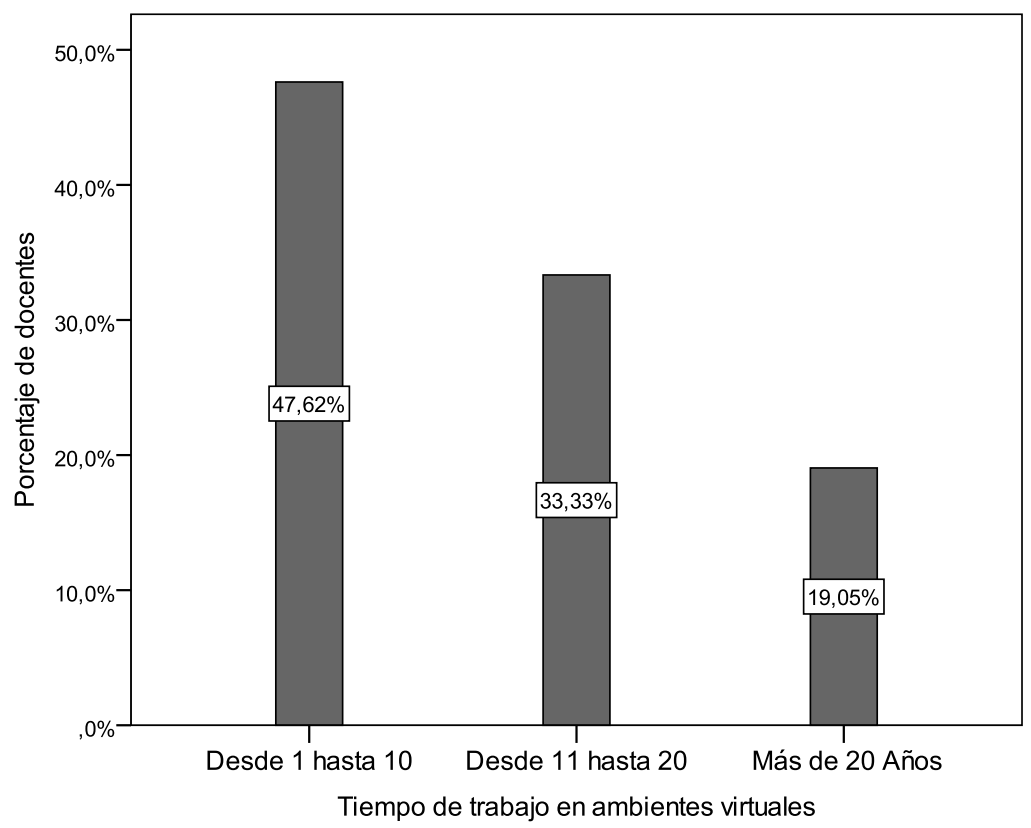

Fig. 2: Tiempo de trabajo del docente en la modalidad virtual en la FESAD.

Adicionalmente, los docentes de la modalidad virtual utilizan algunas veces, las didácticas específicas tradicionales de matemáticas $(52.38 \%)$, sobre ellas, los tutores piensan que son pertinentes para los ambientes interactivos, el $28.57 \%$ casi nunca las requiere y solamente el $19.05 \%$ las usa con frecuencia en la educación virtual (ver Fig. 3). Estos resultados indican de manera moderada que, las didácticas específicas usadas en la enseñanza virtual de las matemáticas, aún no se desligan de las utilizadas en el modelo tradicional; es decir, todavía se acude a estrategias propias de la educación presencial y se transita paulatinamente hacia un modelo opuesto a la frecuente transmisión de información (Martínez et al., 2018). Además, mediante una prueba chi-cuadrado y el coeficiente de contingencia de Pearson se estableció que el tiempo de trabajo en la modalidad virtual y el uso de didácticas específicas tradicionales están moderadamente asociadas aunque de manera no significativa ( $P$ - valor $=0.751$ para las dos pruebas); por lo tanto, las didácticas específicas no convencionales también están asociadas con el tiempo de trabajo del tutor en la modalidad virtual.

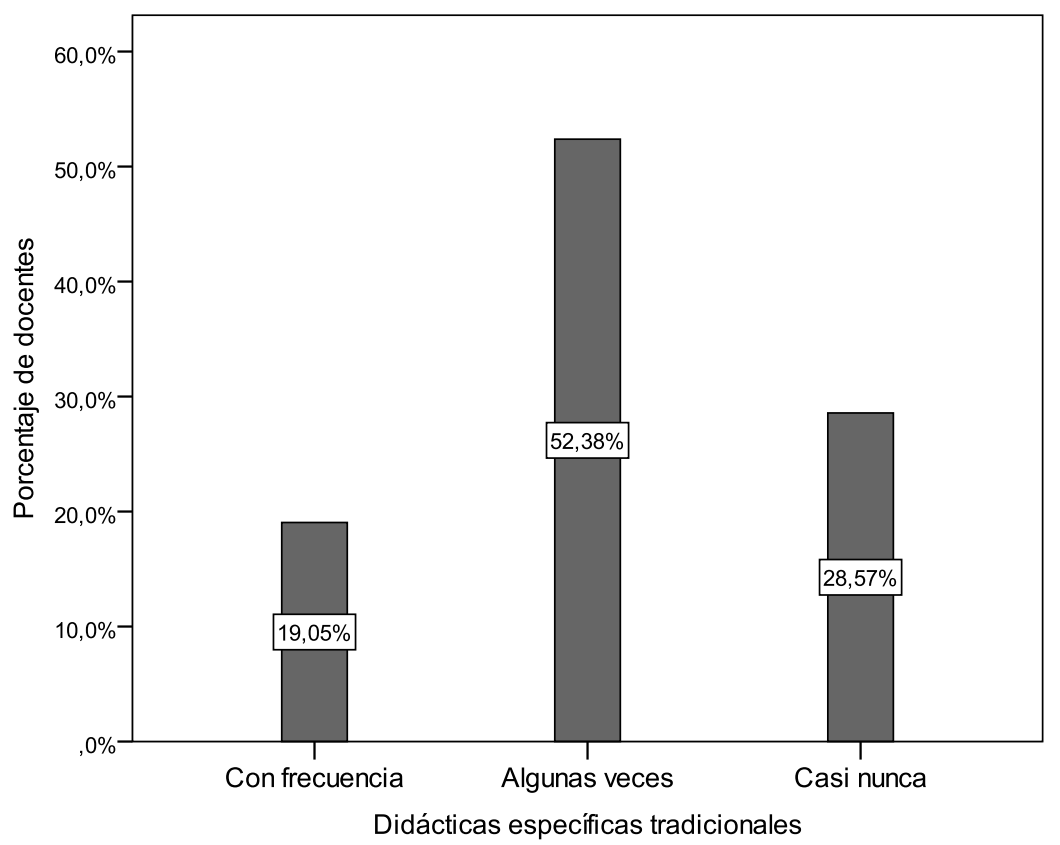

Fig. 3: Uso de didácticas específicas tradicionales en matemáticas en la FESAD

En cuanto a la metodología (ver Fig. 4), el $52.38 \%$ de los docentes se inclina por la constructivista, en un menor porcentaje $(23.81 \%)$ mencionan que utilizan metodologías activas, un $14.29 \%$ aún continua usando la escuela tradicional y un $9.52 \%$ hace uso de otras metodologías (conductistas). Estos porcentajes destacan algunos aspectos característicos del modelo pedagógico empleado por los tutores participantes en este 
estudio, el cual podría considerarse ecléctico. En parte, estos resultados concuerdan con Hernández (2012), quien menciona que, la educación tradicional es partidaria de la enseñanza directa y rígida, predeterminada por un currículo inflexible y centrado en el profesor. García et al. (2018) indican que se puede acudir a una acción tutorial con diferentes enfoques, luego, el conductismo tiene sentido en abordajes de corte técnico y mecanicista, orientados al desarrollo de procesos algorítmicos, en las cuales el sujeto es pasivo y reproductor del conocimiento. Las metodologías activas para enseñar matemáticas están direccionadas a potenciar la participación activa del estudiante en la clase (Marciniak y Sallán, 2018) y la activación de conocimientos previos (Chevallard, 1999), la asimilación de nuevos conceptos y la formalización matemática (Depaepe et al., 2018; Ball et al., 2008), el desarrollo del aprendizaje basado en problemas y en proyectos, el autoaprendizaje, y el aprendizaje tanto cooperativo como colaborativo (Abreu et al., 2018). Finalmente, el docente constructivista actúa como mediador en el proceso enseñanza-aprendizaje de las matemáticas (Marcos, 2009) e impulsa a los estudiantes a descubrir principios por sí mismo y a construir el conocimiento trabajando en la resolución de problemas reales o simulados (Burbano et al., 2015), con frecuencia, en colaboración con otros estudiantes. Esta colaboración también se conoce como proceso social de construcción del conocimiento (Ernest, 1998). El constructivismo como postura epistemológica también se encuentra en la Matemática Educativa y en la aplicación de esta postura en el aula (Miller, 2000).

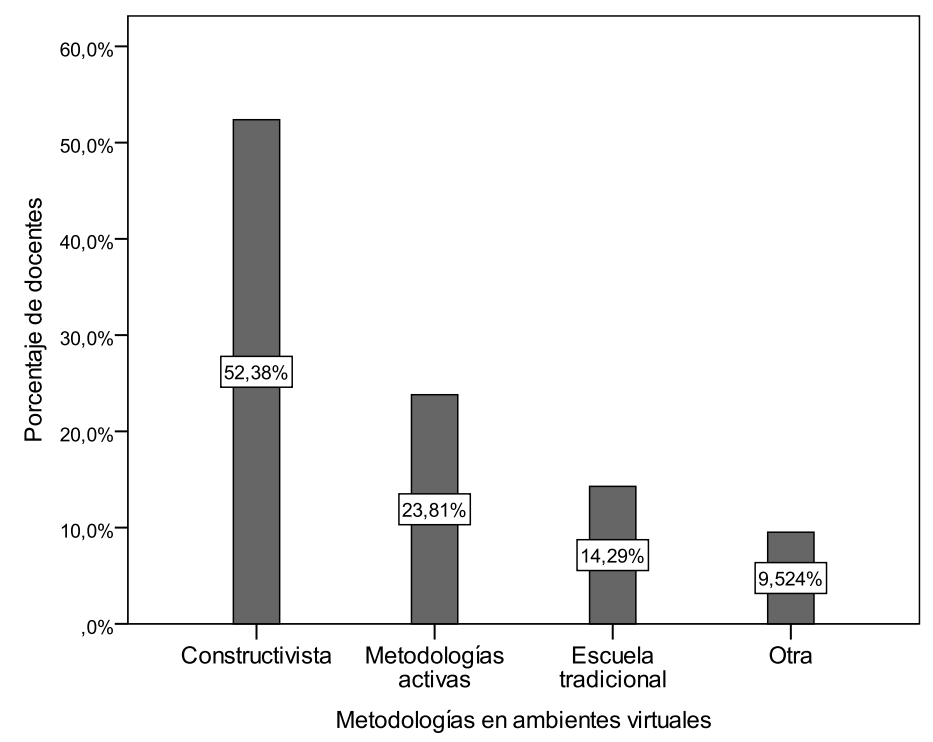

Fig. 4: Metodologías utilizadas por el docente en la enseñanza de las matemáticas en ambientes virtuales.

En referencia a la frecuencia del uso de medios y mediaciones (ver Fig. 5), los encuestados, en un alto porcentaje utilizan todos los relacionados con las TIC $(80.95 \%)$ en sus procesos de enseñanza de las matemáticas (se incluyen: plataforma virtual $(9.52 \%)$, chat (4.76) e internet $(4.76 \%))$. Para orientar sus clases en primera instancia, usan la plataforma virtual (Moodle), la cual también incluye la posibilidad de usar el chat, asimismo, la internet (correo electrónico) y buscadores de información) facilita la comunicación bidireccional entre tutor y estudiantes (García, 1999; Barberá, 2000). Estos aspectos son relevantes en el modelo de educación virtual, al respecto, Castro et al. (2007) indican que este tipo de educación es retadora y potencia el uso de las TIC en nuevas formas de organizar, producir, difundir, acceder y controlar el conocimiento. En conveniente indicar que las herramientas mencionadas son pertinentes para dinamizar los procesos tendientes a enseñar las matemáticas en el modelo de educación virtual.

En la figura 6 , se observa que el $38.1 \%$ de los profesores, se identifican como mediadores en los procesos de enseñanza de las matemáticas; un $23.81 \%$ como docentes, el $19.05 \%$ como orientadores y solamente el $19.05 \%$ se considera como un tutor. Sin embargo, al acumular los porcentajes de las categorías diferentes a la de docente (tradicional), se deduce que el $76.2 \%$ desempeñan el rol de mediador, orientador o tutor, estos aspectos son propios de la educación desarrollada con el modelo virtual. Además, se evidencia que el papel protagónico que toma el profesor es el de mediador, lo cual indica que la figura del docente tradicional ha ido cambiando paulatinamente y ha sido permeada por las TIC, este cambio se hace aún más evidente en el desarrollo de la enseñanza en los nuevos entornos virtuales, que requieren una mediación del aprendizaje distinta a los entornos presenciales. 


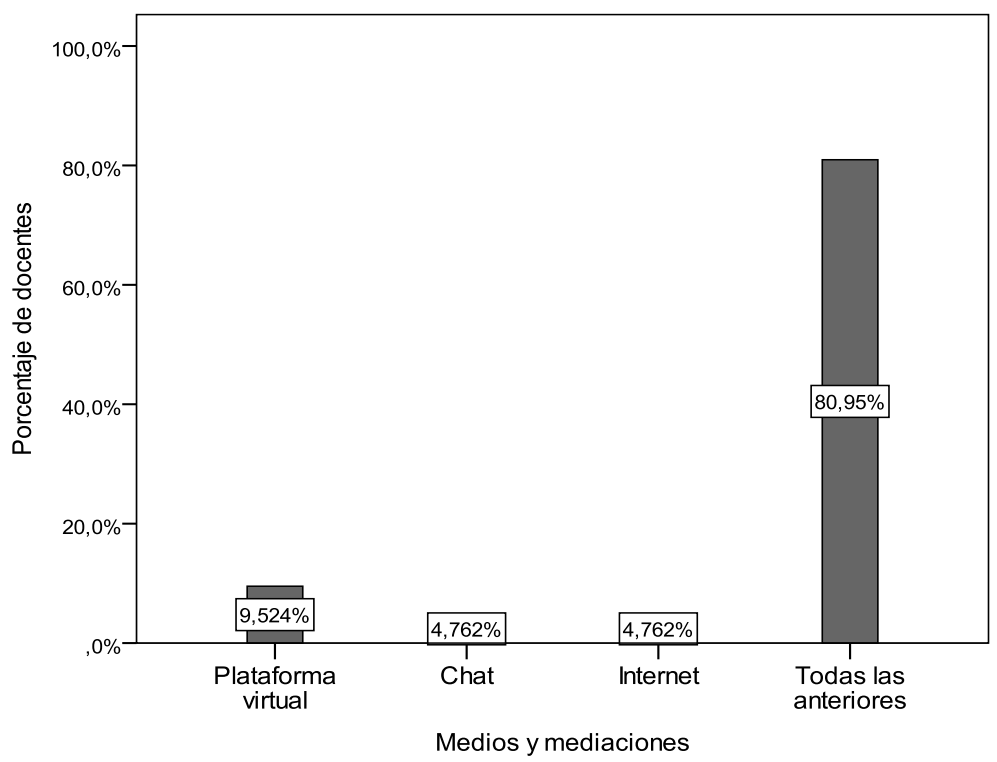

Fig. 5: Medios y mediaciones usados con frecuencia en la enseñanza de las matemáticas en la FESAD.

De la figura 6 se tiene que, el $19 \%$ de los profesores asume el rol de tutor; al parecer, el docente posee capacidad de reflexión para aclarar incertidumbres frente a los diversos interrogantes que se originan en el quehacer académico (Menéndez, 2012). En resumen, estos resultados reflejan que, el papel del tutor se centra en el rol de mediador entre el conocimiento matemático y el estudiante a través de las orientaciones que proporciona a quien aprende en un ambiente virtual; esto guarda relación con lo mencionado por Marcos (2009) respecto al rol del tutor y de García (1999) en referencia a los entornos virtuales.

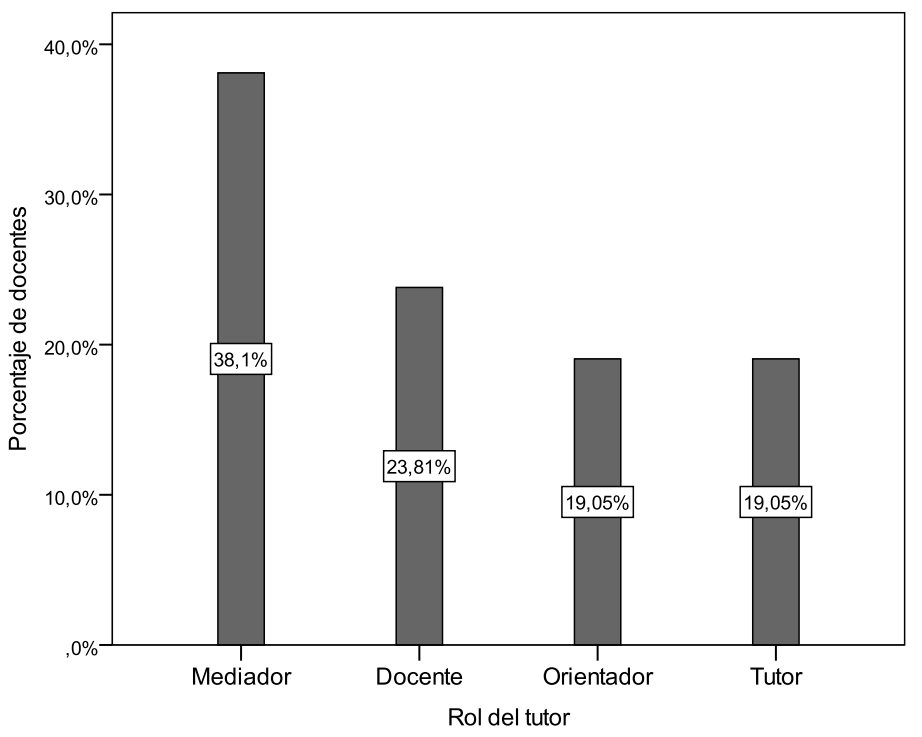

Fig. 6: Rol del tutor durante el proceso de enseñanza de las matemáticas en la FESAD

De la figura 7 , se deduce que el $42.86 \%$ de los profesores indican que la forma más recurrente de evaluar a sus estudiantes es el trabajo independiente seguido por los trabajos en grupo con un $33.33 \%$, el $23.81 \%$ corresponde a evaluaciones escritas y ningún docente usa otras formas de evaluación. Al acumular los porcentajes de las categorías de trabajo independiente y de trabajos en grupo, se establece que, el $76.19 \%$ de los tutores emplean formas de evaluación propias del modelo de educación virtual centrada en el trabajo colaborativo que se hace a través de la plataforma; estas formas de evaluación se asocian con lo señalado por García (1999), quien asocia el modelo de educación virtual con un sistema tecnológico complejo de acción tutorial que también propicia la evaluación del aprendizaje.

El trabajo independiente forma parte del aprendizaje autónomo centrado en el estudiante que, se incluye en esta modalidad y atiende a las características propias del alumnado, tales como su individualidad, particularidades, debilidades y fortalezas. No obstante, estas tres formas de evaluación no son suficientes en el modelo virtual, el tutor también ha de realizar una evaluación formativa tendiente a mejorar la motivación, la empatía, la autoestima estudiantil y la interacción grupal (García et al., 2018) que coadyuven con su 
interacción y comprensión de las matemáticas. Además, por medio de una prueba chi-cuadrado y el coeficiente de contingencia se determinó que, las metodologías y las formas de evaluación se asocian de manera moderada, aunque la relación es no significativa ( $P$ - valor $=0.349$ para las dos pruebas); de forma semejante, las didácticas específicas y la evaluación ( $P$-valor $=0.253)$ también se asocian moderadamente.

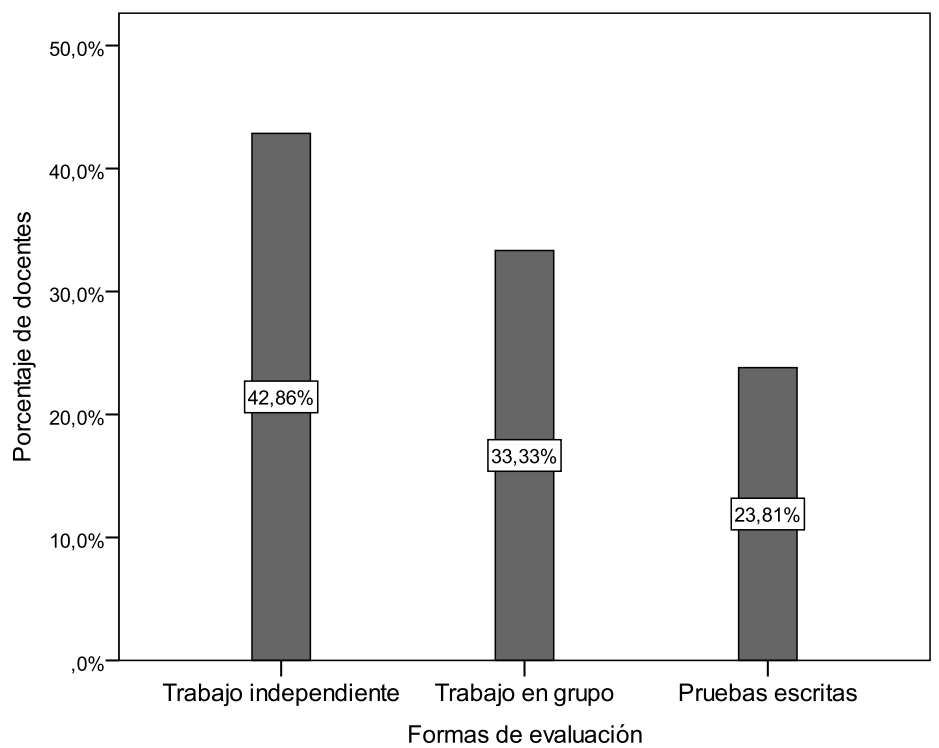

Fig. 7: Formas de evaluación en la enseñanza de las matemáticas en la FESAD.

De manera general, al culminar este estudio, los resultados revelan que, el tutor que enseña matemáticas en el programa de ciencias tecnológicas de la FESAD, se caracteriza por poseer un alto nivel de conocimiento matemático producto de su formación profesional, con una amplia experiencia docente, quienes además, en un $52.38 \%$ presentan más de 10 años de trabajo en la modalidad virtual y paulatinamente han involucrado didácticas específicas de la matemática, de manera no convencional, en ambiente virtual; también, favorecen la construcción del conocimiento matemático mediante el uso de metodologías activas; en un alto porcentaje, se apoyan en medios y mediaciones asociadas con las TIC y centran su evaluación en el trabajo independiente y en grupo prioritariamente. Estos resultados contrastan con los obtenidos por Martínez et al. (2018), quienes concluyen que un alto porcentaje de los docentes que participaron en su estudio, estaban preparados para desempeñarse de forma adecuada en los procesos educativos virtuales; establecieron que, un $52.7 \%$ de los tutores tenían un alto nivel de dominio conceptual y de estrategias pedagógicas propias de la educación virtual, seguidos de un $45.5 \%$ con un nivel moderado y de un $1.8 \%$ con nivel bajo.

\section{CONCLUSIONES}

De acuerdo al trabajo presentado y a los resultados obtenidos, se pueden platear las siguientes conclusiones referidas al tutor de matemáticas que trabaja en ciencias tecnológicas de la FESAD: 1) Moderadamente se aproxima al requerido en el modelo de educación virtual; su fortaleza radica en su conocimiento disciplinar, utiliza algunas herramientas virtuales y estrategias asociadas con las didácticas específicas, que con alguna frecuencia fueron adaptadas desde la enseñanza tradicional de las matemáticas. 2) Sus didácticas específicas están moderadamente asociadas con su tiempo de trabajo en la modalidad virtual; algunas veces, recurre al uso de las que comúnmente se utilizan en la enseñanza tradicional de las matemáticas, tanto a nivel universitario como de la educación secundaria. 3) Su experiencia profesional docente y tiempo de trabajo en la modalidad virtual, han influido de manera proactiva en la búsqueda de estrategias alternativas para mejorar la enseñanza de las matemáticas, mediante el uso frecuente de medios tecnológicos. 4) Sus metodologías y formas de evaluación están moderadamente asociadas y sí guardan relación con el rol del tutor en el modelo de educación virtual en la enseñanza de las matemáticas. 5) Usa el trabajo independiente y en grupo, como formas prioritarias de evaluación destinadas a valorar los aprendizajes de los estudiantes.

\section{REFERENCIAS}

Abreu, O., S. Rhea, G. Arciniegas y M. Rosero, Objeto de Estudio de la Didáctica: Análisis Histórico Epistemológico y Crítico del Concepto, doi: 10.4067/S0718-50062018000600075, Formación Universitaria, 11(6), 75-82 (2018)

Ball, D.L., M.H. Thames y G. Phelps, Content knowledge for teaching: What makes it special? doi: 10.1177/0022487108324554, Journal of Teacher Education, 59(5), 389-407 (2008)

Barberá, E., Study actions in a virtual university, Virtual University Journal, 3(2), 31-42 (2000) 
Brousseau, G., Fondements et méthodes de la didactiques des mathématiques, Recherches en Didactique des Mathématiques, 7(2), 33-115 (1986)

Burbano, V.M.A., M.A. Valdivieso y E. Aldana, Conocimiento base para la enseñanza: un marco aplicable en la didáctica de la probabilidad, Revista de Investigación, Desarrollo e Innovación, 7(2), 269-285 (2017)

Burbano, V.M., J.E. Pinto y M.A. Valdivieso, Formas de usar la simulación como un recurso didáctico, Revista Virtual Universidad Católica del Norte, 45, 16-37 (2015)

Castro, S., G. Belkys y D. Casado, Las TIC en los procesos de enseñanza y aprendizaje, Laurus, 13(23), 213-234 (2007)

ChanLin, L.J., Students' Involvement and Communit Support for Service Engagement in Online Tutoring, Journal of Educational Media y Library Sciences, 53(2), 245-268 (2016)

Chevallard, Y., L'analyse des pratiques enseignantes en théorie anthropologique du didactique, Recherches en Didactique des Mathématiques, 19(2), 221-266 (1999)

Colestock, A. y M. Sherin, Teachers' sense-making strategies while watching Video of mathematics instruction, Journal of Technology and Teacher Education, 17(1), 7-29 (2009)

D’Amore, B. y J.D. Godino, Punti di vista antropologico ed ontosemiotico, Didattica della Matematica, 20(1), 9-38 (2006)

Depaepe, F., L. Verschaffel y G. Kelchtermans, Pedagogical content knowledge: A systematic review of the way in which the concept has pervaded mathematics educational research, Teaching and Teacher Education, 34, 12-25 (2013)

Ernest, P., Social Constructivism as a Philosophy of Mathematics, Albany, NY: State University of New York Press (1998)

Fraser, S.P., Pedagogical Content Knowledge (PCK): Exploring its Usefulness for Science Lecturers in Higher Education, Research in Science Education, 1-21 (2015)

García, L., Historia de la Educación a Distancia (History of Distance Education), Revista Iberoamericana de Educación a Distancia, 2(1), 11-40 (1999)

García, S.A., A.M. Rodríguez y M.P.C. Reche, Análisis de la Acción Tutorial y su Incidencia en el Desarrollo Integral del Alumnado. El Caso de la Universidad de Castilla La Mancha, España, doi: 10.4067/S0718-50062018000300063, Formación Universitaria, 11(3), 63-72 (2018)

Hernández, M., Modelos tutoriales en la educación a distancia a través de la tecnología de la información y de la comunicación: tarea del docente tutor. Tesis Doctoral no publicada, Universidad Nacional de Educación a Distancia, Madrid, España (2012)

Hernández, S., C. Fernández y L. Baptista, Metodología de la Investigación, McGraw-Hill, 3 Ed., México (2003)

Marciniak, R. y J.G. Sallán, Dimensiones de evaluación de calidad de educación virtual: revisión de modelos referentes, RIED, Revista Iberoamericana de Educación a Distancia, 21(1), 217-238 (2018)

Marcos, G., Un modelo de análisis de competencias matemáticas en un entorno interactivo, Colección Digital Eudoxus, 9, (2009)

Martínez, O., E.J. Steffens, D.C. Ojeda y H.G. Hernández, Estrategias pedagógicas aplicadas a la educación con mediación virtual para la generación del conocimiento global, doi: 10.4067/S0718-50062018000500011, Formación Universitaria, 11(5), 11-18 (2018)

Mason, J., Researching your own practice: The discipline of noticing, Routledge, London (2002)

Menéndez, C., Mediadores y mediadoras del aprendizaje, Competencias docentes en los entornos virtuales de aprendizaje, Revista Iberoamericana de Educación, (60), 39-50 (2012)

Miller, J.B., The quest for the constructivist statistics classroom: viewing practice through constructivist theory, Tesis Doctoral no publicada, Universidad de Ohio (2000)

Pagano, C.M., Los tutores en la educación a distancia. Un aporte teórico; Barcelona: Universidad Oberta de Catalunya, Revista de Universidad y Sociedad del Conocimiento, 3-17 (2008)

Schoenfeld, A.H. y J. Kilpatrick, Toward a theory of proficiency in teaching mathematics. In D. Tirosh y T. Wood (Eds.), Tools and processes in mathematics teacher education, 321-354, Sense Publishers, Rotterdam, Holanda (2008)

Shulman, L., Those who understand: knowledge growth in teaching, Educational Researcher, 15(2), 4-14 (1986)

Silva, J., El rol del tutor en los entornos virtuales de aprendizaje, Innovación Educativa, ISSN: 1665-2673, 10(52), 13-23, Instituto Politécnico Nacional Distrito Federal, México (2010)

Stein, M. K., J. Remillard y M. Smith, How curriculum influences student learning. In F. Lester (Ed.), Second handbook of mathematics teaching and learning, 319-369, Greenwich, CT, Information Age (2007)

Villalobos, A.A. e Y.M. Melo, Narrativas Docentes como Recurso para la Comprensión de la Transferencia Didáctica del Profesor Universitario, doi: 10.4067/S0718-50062019000100121, Formación universitaria, 12(1), 121-132 (2019) 